\title{
Há equivalência entre a temperatura da membrana timpânica e a temperatura retal em ovinos Santa Inês normotérmicos?
}

\author{
Is there equivalence between the tympanic membrane temperature and the rectal temperature \\ in normothermic Santa Ines sheep?
}

Claudia Del CampoI ${ }^{I}$ Vanner Boere ${ }^{\text {II }}$

\begin{abstract}
A utilização do termômetro de raios infravermelhos para a aferição da temperatura timpânica em animais tem se tornado uma alternativa prática na clínica veterinária. Em ovinos, a mensuração da temperatura timpânica não foi suficientemente comparada com a aferição da temperatura retal. Este estudo avaliou a equivalência entre a temperatura timpânica e a temperatura retal em 16 ovinos da raça Santa Inês. Durante a coleta de dados, a temperatura e a umidade relativa do ar foram monitoradas e não houve correlação entre estas e a temperatura corporal. A análise da equivalência, pelo método da diferença entre as temperaturas, resultou em temperaturas timpânicas mais baixas (entre 35 e 36,9 $9^{\circ} \mathrm{C}$, para ambos os ouvidos) que a temperatura retal (entre 39 e $38^{\circ} \mathrm{C}$ ). Os limites de concordância das comparações entre a temperatura retal e as temperaturas timpânicas foram acima do valor pré-estabelecido. A localização anatômica dos locais aferidos e o tipo de termômetro contribuíram para as diferenças de temperatura. A temperatura retal e a temperatura timpânica foram independentes, sugerindo não haver equivalência entre ambas.
\end{abstract}

Palavras-chave: ovinos, temperatura timpânica, temperatura retal, Santa Inês, termômetro.

\section{ABSTRACT}

The use of infrared thermometers to obtain the tympanic temperature in animals has become a convenient alternative in veterinarian medicine practice. In sheep, tympanic and rectal temperatures have not yet been compared enough. This study evaluated the equivalence between the tympanic and the rectal temperatures in sixteen Santa Ines sheep. During data collection, air temperature and humidity were monitored and there was no discernible correlation between these and the ovine body temperature. The analysis of the equivalence, as measured by the method of the difference amongst temperatures, resulted in lower tympanic temperature (between 35 and $36.9^{\circ} \mathrm{C}$, for both ears) than the rectal temperature (between 38 and $39^{\circ} \mathrm{C}$ ). The agreement limit of the comparisons between the rectal and tympanic temperatures was above values established previously in the literature. The anatomical site used for the temperature measurement and the type of thermometer used contributed to these temperature differences. The rectal and tympanic temperatures were independent, suggesting that there is no equivalence between them.

Key words: sheep, tympanic temperature, rectal temperature, Santa Ines, thermometer.

\section{INTRODUÇÃO}

A eficácia da medida da temperatura retal (TR) pode ser comprometida por presença de fezes no reto, peristaltismo aumentado e posicionamento incorreto do termômetro (KUNKLE et al., 2004). A TR é influenciada pela atividade ruminal e pelo ambiente (BERMAN, 1971). Outro problema está relacionado à pouca fidelidade das alterações térmicas que estão ocorrendo em órgãos vitais como o cérebro (MALONEY et al., 2001). Alternativamente, a mensuração da temperatura da membrana timpânica pode ser mais rápida e precisa, por isso está sendo usada mais freqüentemente na clínica veterinária. Em ovinos, a mensuração da temperatura timpânica não foi suficientemente comparada com a

\footnotetext{
'Programa de Pós-graduação em Ciências Agrárias, Faculdade de Agronomia e Veterinária, Universidade de Brasília (UnB), Brasília, DF, Brasil.

IIDepartamento de Ciências Fisiológicas, Instituto de Biologia, UnB, 70910-900, Brasília, DF, Brasil. Email: vanner@unb.br. Autor para correspondência.
} 
aferição da TR. Não existe um termômetro timpânico exclusivo para pequenos ruminantes. O modelo existente foi aprovado para cães, gatos, primatas, cobaias e chinchilas (REXROAT et al., 1999). Este estudo teve o objetivo de verificar a equivalência entre a temperatura timpânica e a retal em ovinos Santa Inês.

Foram utilizados 16 ovinos adultos (dois machos e 14 fêmeas), da raça Santa Inês, sem sinais clínicos de alguma patologia. Os ovinos eram criados de forma semi-extensiva em pasto misto e abrigados em galpão à noite. As aferições ocorreram duas vezes em um intervalo de 14 dias, às $7 \mathrm{~h}$, antes da soltura no campo. Os animais eram contidos gentilmente antes da saída de um galpão de abrigo noturno. A cabeça do ovino era posicionada para a aferição da temperatura timpânica direita (TTD) e esquerda (TTE) em ordem aleatória. As TTD e TTE foram aferidas com termômetro timpânico veterinário de raios infravermelhos (Pet-Temp Ear Thermometer - Advanced Monitors - EUA). O aparato foi posicionado no meato auditivo de forma a seguir a forma do canal e evitar o contato do sensor térmico com a derme. A leitura era realizada em um segundo. A temperatura retal foi aferida com um termômetro digital de haste flexível (Pro-Check - ONBO Eletronics, EUA) inserido cerca de cinco centímetros no reto. Em aproximadamente 90 s a leitura era concluída. A temperatura do ambiente foi monitorada por meio de um termohigrômetro (Extech, China).

A análise da equivalência entre a temperatura timpânica e a temperatura retal seguiu o método da diferença entre a temperatura retal pela temperatura timpânica (KUNKLE et al., 2004). Neste método calcula-se a média e o desvio padrão (DP), seguido dos limites de concordância (isto é, média $\pm 1,96$ DP) entre as medidas nas duas partes do corpo (KUNKLE et al., 2004). Uma diferença maior que $\pm 0,49^{\circ} \mathrm{C}$, baseada nos resultados de outro estudo com ovinos Santa Inês na região do Distrito Federal (QUESADA et al., 2001), foi considerada fora da amplitude clinicamente aceitável. Em outras palavras, quando a diferença foi maior que $0,49^{\circ} \mathrm{C}$, para mais ou para menos, concluiu-se que as temperaturas não foram equivalentes e não poderiam ser interpretadas como dependentes para uma avaliação clínica da temperatura corporal central.

O limite superior para temperatura retal para ovinos Santa Inês normotérmicos é 38,99 ${ }^{\circ} \mathrm{C}$ (QUESADA et al., 2001). Para a temperatura timpânica, as referências são raras, mas um estudo com ovinos selvagens (Ovis canadensis) registrou $35,7^{\circ} \mathrm{C}$ como limite inferior sob esforço de fuga e contenção (DREW, 1996). Para evitar a sobreposição de índices térmicos de hipotermia, hipertermia e febre, eliminaram-se da amostra pareamentos com qualquer temperatura abaixo de $35,0^{\circ} \mathrm{C}$ ou acima de $39,0^{\circ} \mathrm{C}$, resultando em um descarte de 14 aferições das 32 coletadas.

A temperatura retal máxima e mínima foi 39,0 e $38,0^{\circ} \mathrm{C}$, respectivamente. Na membrana timpânica esquerda, a temperatura máxima foi 36,9 e a mínima foi $35,1^{\circ} \mathrm{C}$. A temperatura máxima da membrana timpânica direita foi 36,7 e a mínima foi $35,0^{\circ} \mathrm{C}$.

A média da diferença entre a temperatura retal e a temperatura timpânica esquerda (TR-TTE) foi $2,92 \pm 0,66^{\circ} \mathrm{C}$. A máxima diferença foi 3,8 e a mínima diferença foi $1,8^{\circ} \mathrm{C}$, podendo ser interpretado que a TTE esteve abaixo da TR. O intervalo de confiança a 95\% situou-se entre $2,26 \mathrm{e} 3,58^{\circ} \mathrm{C}$. Os limites de concordância foram 1,62 e $4,20^{\circ} \mathrm{C}$.

A média da diferença entre a temperatura retal e a temperatura timpânica direita (TR-TTD) foi $3,0 \pm 0,6^{\circ} \mathrm{C}$. A máxima diferença foi 3,8 e a mínima diferença foi $2,0^{\circ} \mathrm{C}$. O intervalo de confiança a $95 \%$ situou-se entre 2,4 e $3,6^{\circ} \mathrm{C}$. Com base nesses valores, pode-se interpretar que a TTD esteve abaixo da TR. Os limites de concordância foram 1,82 e 4,17 ${ }^{\circ} \mathrm{C}$.

Os limites de concordância das comparações entre a TR e a TTE e a TR e a TTD foram muito acima dos limites de concordância pré-estabelecidos para este estudo $\left( \pm 0,49^{\circ} \mathrm{C}\right)$, como requisito para uma equivalência entre as aferições retais e timpânicas. A temperatura do ambiente (amplitude de 17,0 a $18,7^{\circ} \mathrm{C}$ ) não se correlacionou com a TR $(r=0,22$; $\mathrm{p}=0,78)$, com a TTE $(r=-0,12 ; \mathrm{p}=0,37)$ ou com a TTD $(r=-0,10 ; \mathrm{p}=0,44)$.

As temperaturas timpânicas estiveram mais baixas do que as temperaturas retais em concordância com outros estudos (LABURN et al., 1988; MALONEY et al., 2001; MARAÍ et al., 2007). A explicação para estas diferenças pode ser atribuída às propriedades intrínsecas das regiões aferidas e menos provavelmente aos aparelhos utilizados.

A maior TR poderia se dever em parte à influência do clima, mas não ocorreu uma dependência entre os valores da temperatura ambiente e as temperaturas mensuradas. Particularmente a TR é fortemente influenciada pela temperatura do rúmen (MARAÍ et al., 2007) devido ao peristaltismo e à ação da microbiota ruminal.

Por outro lado, a temperatura da membrana timpânica depende de tributárias da carótida interna, que irriga o cérebro. A rede carotídea (LABURN et al., 1988) capacita os ovinos para "resfriarem" o sangue antes que este adentre o cérebro (CAPUTA, 2004). Outro mecanismo que auxilia no resfriamento da temperatura cerebral e da membrana timpânica é o relaxamento das arteríolas por ação reflexa do dióxido de carbono $\left(\mathrm{CO}_{2}\right)$ e do óxido nítrico (NO), potentes 
vasodilatadores (MEINERS \& DABBS, 1977; DREVETS \& RAICHLE, 1995). A temperatura timpânica parece ser influenciada pela temperatura cerebral, podendo servir como um parâmetro sobre a condição térmica central (CHERBUIN \& BRINKMAN, 2007).

Em ovelhas deslanadas como a raça Santa Inês, a perda de calor pode ser mais acentuada, conferindo temperaturas baixas na região cerebral e nos tímpanos. A conjunção de mecanismos que aumentam a temperatura retal e de mecanismos que resfriam o cérebro e, por conseguinte, a temperatura timpânica pode conferir diferenças acentuadas entre a região timpânica e outras regiões do corpo.

Os diferentes aparelhos e as técnicas de aferição podem ser a outra explicação para as diferenças encontradas. $\mathrm{O}$ termômetro clínico digital de haste e o termômetro timpânico dependem de um posicionamento adequado do sensor. No caso deste estudo, as aferições foram realizadas pelo mesmo pesquisador, com grande experiência clínica, tornando o erro por manipulação pouco provável.

A falta de equivalência entre os dois tipos de aparelhos, nas regiões citadas, já fora observada em gatos (KUNKLE et al., 2004), humanos (GREENES \& FLEISCHER, 2004) e primatas não-humanos (BOERE et al., 2003; SHELTON et al., 2006). Os valores térmicos de referência (QUESADA et al., 2001) e a estreita amplitude térmica do ambiente permitiram que a comparação entre as regiões e a avaliação dos aparelhos fosse confiável, excluídas as variações cronobiológicas ou os extremos térmicos ambientais.

Há necessidade de mais dados com ovinos em circunstâncias de estresse térmico e febre para verificar a equivalência dos valores térmicos retais e timpânicos com os dois aparelhos. Os dados de outros estudos, no entanto, demonstraram que quanto maior a temperatura retal maior será a diferença em relação à temperatura cerebral ou timpânica em ovinos (MALONEY et al., 2001). Em suma, os valores térmicos aferidos na membrana timpânica e no reto, por estas diferentes técnicas, não podem ser clinicamente interpretados como equivalentes.

\section{AGRADECIMENTOS}

Somos gratos a Christian Hoffmann pela inestimável ajuda na apreciação da partes deste texto e a João Paulo Gravina pela coleta parcial de dados.

\section{REFERÊNCIAS}

BERMAN, A. Thermoregulation in intensively lactating cows in near-natural conditions. Journal of Physiology, Oxford, v.215, p.477-489, 1971
BOERE, V. et al. Correlation between tympanic and rectal temperature in marmosets (Callithrix penicillata) under acute stress. Brazilian Journal of Veterinary Research and Animal Science, São Paulo, v.40, p.90-95, 2003.

CAPUTA, M. Selective brain cooling: a multiple regulatory mechanism. Journal of Thermal Biology, Amsterdam, v.29, p.691-702, 2004.

CHERBUIN, N.; BRINKMAN, C. Sensitivity of functional tympanic membrane thermometry (fTMT) as an index of hemispheric activation in cognition. Laterality, Oxfordshire, v.12, n.3, p.239-261, 2007.

DREVETS, W.C.; RAICHLE, M.E. Positron emission tomographic imaging studies of human emotional disorders. In: GAZZANIGA, M.S. (Ed.). The cognitive neurosciences, Cambridge, Massachusetts: The Mit, 1995. p.1153-1164.

DREW, M.L. The use of a tympanic membrane thermometer for assessing hyperthermia in bighorn sheep. Journal of Wildlife Diseases, Stanford, v.32, n.3, p.512-516, 1996.

GREENES, D.S.; FLEISCHER, G.R. When body temperature changes, does rectal temperature lag? Journal of Pediatrycs, New York, v.144, p.824-826, 2004.

KUNKLE, G.A. et al. Comparison of body temperature in cats using veterinary infrared thermometer and a digital rectal thermometer. Journal of American Animal Hospital Association, Stanford, v.40, p.42-46, 2004

LABURN, H.P. et al. Effects of tracheostomy breathing on brain and body temperatures in hyperthermic sheep. Journal of Physiology, Oxford,v.406, p.331-344, 1988.

MALONEY, S.K. et al. Rectal temperature measurement results in artifactual evidence of selective brain cooling. American Journal of Physiology: Regulatory, Integrative, Comparative Physiology, Stanford, v.281, p.R108-R114, 2001.

MARAÍ, I.F.M.et al. Physiological traits as affected by heat stress in sheep - a review. Small Ruminant Research, Amsterdam, v.71, n.1-3, p.1-22, 2007.

MEINERS, M.L.; DABBS, J.M. Ear temperature and brain blood flow: laterality effects. Bulletin Psychonomic Society, Austin, v.10, p.194-196, 1977.

QUESADA, M. et al. Tolerância ao calor de duas raças de ovinos deslanados no Distrito Federal. Revista Brasileira de Zootecnia, Viçosa, v.30, n.3, p.1021-1026, 2001.

REXROAT, J. et al. Clinical accuracy of Vet-Temp instant ear thermometer: comparative study with dogs and cats. San Diego: Advances Monitors Corporation, 1999. p.1-4.

SHELTON JR, L.J. et al. A comparison of non-contact, subcutaneous, and rectal temperatures in captive owl monkeys (Aotus sp). Journal of Medical Primatology, Oxford, v.35, p.346-351, 2006. 